\title{
Disrupted interhemispheric functional coordination in patients with chronic low back-related leg pain: a multiscale frequency-related homotopic connectivity study
}

This article was published in the following Dove Press journal:

Journal of Pain Research

\author{
Yong Zhang' \\ Yanyan Zhu ${ }^{2,3}$ \\ Yixiu $\mathrm{Pei}^{2,3}$ \\ Yanlin Zhao ${ }^{2,3}$ \\ Fuqing Zhou ${ }^{2,3}$ \\ Muhua Huang ${ }^{2,3}$ \\ Lin $\mathrm{Wu}^{2,3}$ \\ Daying Zhang' \\ Honghan Gong ${ }^{2,3}$ \\ 'Department of Pain Clinic, The First \\ Affiliated Hospital, Nanchang University, \\ Nanchang, Jiangxi Province 330006, \\ People's Republic of China; ${ }^{2}$ Department \\ of Radiology, The First Affiliated Hospital, \\ Nanchang University, Nanchang 330006, \\ People's Republic of China; \\ ${ }^{3}$ Neuroradiology Lab, Jiangxi Province \\ Medical Imaging Research Institute, \\ Nanchang 330006, People's Republic of \\ China
}

\begin{abstract}
Objective: Chronic low back pain has been observed to decrease movement coordination. However, it is unclear whether the existing alteration of inter-hemispheric synchrony of intrinsic activity in patients with chronic low back-related leg pain (cLBLP). The present study aims to investigate the alteration of homotopic connectivity and its clinical association with the cLBLP patients.

Participants and methods: A cohort of cLBLP patients $(n=25)$ and well-matched healthy controls $(\mathrm{HCs})(\mathrm{n}=27)$ were recruited and underwent MRI scanning and a battery of clinical tests. The voxel-mirrored homotopic connectivity (VMHC) was used to analyze the interhemispheric coordination in the typical $(0.01-0.1 \mathrm{~Hz})$ as well as five specific (slow- 6 to slow-2) frequency bands and associated with clinical index in cLBLP patients.
\end{abstract}

Results: We observed that cLBLP patients with lower homotopic connectivity than HCs in the inferior temporal gyrus, the superior temporal gyrus, the basal ganglia, the middle frontal gyrus, and the medial prefrontal cortex in the typical and five specific frequency bands, respectively. In the typical and five specific frequency bands, significant positive correlations were observed between the VMHC values of medial prefrontal cortex and the visual analogue scale scores, while the VMHC values of basal ganglia negative correlated with the values of two-point tactile discrimination (2PD) test for the right hand in cLBLP patients, etc. Further receiver operating characteristic curve analysis revealed that VMHC in the above regions with decreased could be used to differentiate the cerebral functional plasticity of cLBLP from healthy individuals with high sensitivity and specificity.

Conclusion: Our results imply that multiscale frequency-related interhemispheric disconnectivity may underlie the central pathogenesis of functional coordination in patients with cLBLP.

Keywords: interhemispheric integration, voxel-mirrored homotopic connectivity, chronic low back-related leg pain, chronic pain, resting-state fMRI

Correspondence: Fuqing Zhou

Department of Radiology, The First Affiliated Hospital, Nanchang University,

17 Yongwaizheng Street, Nanchang, Jiangxi 330006, People's Republic of China Tel +867918 8695132

Emailff.chou@yahoo.com

Daying Zhang

Department of Pain Clinic, The First

Affiliated Hospital, Nanchang University,

17 Yongwaizheng Street, Nanchang,

Jiangxi 330006, People's Republic of China

Tel +867918 8693825

Email dyzsino@ncu.edu.cn

\section{Introduction}

Low back pain is an extremely common acute episode and/or chronic disorder, it affects millions of individuals each year on a world scale. ${ }^{1}$ In fact, approximately two-thirds of patients with low back pain also report leg pain (involving the nerve root), so-called, low back-related leg pain (LBLP). Previous studies observed a relationship between the increased disability and pain, poorer quality of life, poor 
outcomes, as well as lower functional status in patients with chronic LBLP (cLBLP), when compared to the patient with simplicity low back pain. ${ }^{2-4}$ In fact, including in chronic low back pain and cLBLP patients, sensation and/or movement coordination impairment was been observed and discussed as one of the possible mechanisms. ${ }^{3,5,6}$ Although it is unclear, it is reasonable to believe that neural plasticity or rewiring of the brain might play a role in sensation and/or movement coordination of LBLP patients.

It is not altogether without reason to say so. Because in recently, functional MRI studies showed that widespread abnormalities of intrinsic oscillation amplitude ${ }^{7}$ and local connectivity $^{8}$ in the pain matrix (including somatosensory and motor cortices) and information-processing regions and the default mode network in cLBLP patients. Moreover, in chronic pain or chronic low back pain, morphometry and structural studies also demonstrated that significant altered neuronal-glial interactions in the somatosensory cortex, ${ }^{9}$ inconsistent cortical atrophy ${ }^{10,11}$ or thinning ${ }^{12}$ in the sensorimotor, medial and lateral frontal white matter structural damage, ${ }^{13-15}$ which provide an anatomy foundation in understanding the functional alterations.

Right now, the challenge is how to comprehensive understand functional coordination related coding of interhemispheric activity in patients with cLBLP, despite the apparent lack of ongoing straight evidence of structural damage in the corpus callosum. Voxel-mirrored homotopic connectivity (VMHC) is a data-driven method of restingstate functional magnetic resonance imaging (rs-fMRI), by computing the connectivity between each voxel in one hemisphere and its mirrored counterpart, designed to quantify an important feature of the brain intrinsic functional architecture. ${ }^{16,17}$ Homotopic connectivity was conducted to reflecting the importance of interhemispheric communication with the integration of brain functions, as a reliable and reproducible rs-fMRI metric. ${ }^{16,18}$ Recently studies have observed that the status of interhemispheric connectivity is associated with pain sensitivity, ${ }^{19}$ somatosensory neuroplasticity in chronic pain, ${ }^{20}$ and rehabilitation treatment of post-stroke pain. ${ }^{21}$

On the other hand, recent studies have shown that connectivity within specific low-frequency ranges was linked with a variety of neural processes, including input selection, binding, and the cyclic modulation. ${ }^{22-25}$ Experimental animal investigations confirmed that increased rhythmic oscillatory astrocyte activity in neuronal firing is associated with neuropathic pain. ${ }^{26,27}$ Thus, it is conceivable that, in individuals with cLBLP, to explore the homotopic connectivity and it's an association in pain conditions, frequency bands should be considered.

In this study, we hypothesize that the disconnection of interhemispheric in patients with cLBLP, hinting a decrease of the coordination or integration ability of left and right cerebral hemisphere. Herein, we employee VMHC in different frequency subbands (for frequency property) to examined extract the neurophysiological basis of interhemispheric connectivity in cLBLP patients at resting state, and associated with disease severity or others index. Also, we used a seed-based functional connectivity analysis to reveal the heterotopic connectivity patterns of the regions with altered VMHC in patients, because homotopic connectivity might influence its specific networks.

\section{Materials and methods}

\section{Participants}

At first, two groups of participants were recruited for this study, including 33 right-handed cLBLP patients and age-, gender- and education-matched healthy controls (HCs). All the subjects were recruited from our hospital and the local community from Oct. 2016 to Jan. 2018. The LBLP patients were including criterion as follows: (1) clinical and radiology diagnosed with clear evidence of discogenic compression on a lumbar CT and/or MRI, when available (For example, Figure 1); (2) chronic (more than 12 weeks) radiating pain the buttock(s) and lower limb(s); (3) Visual analogue scale (VAS) scores of all patients were above 4 (moderate to severe pain); (4) failed to respond to conservative treatment of medications, e.g., anti-inflammatory drugs (Motrin, Advil and Naproxen) and acetaminophen (e.g., Tylenol) without opioids, exercise and physical therapy. ${ }^{28}$ The cLBLP and HCs were also excluded (1) if they reported a major systemic disease or history of head and spinal cord injury; (2) spinal stenosis due to calcifications on the spinal protrusion, lateral recess stenosis, spinal stenosis, pyriformis syndrome, or sciatica.

This study was approved and performed following the guidelines of the Medical Research Ethics Committee and the Institutional Review Board of the First Affiliated Hospital, Nanchang University, China, and following the Declaration of Helsinki (2000). All patients and HCs give 


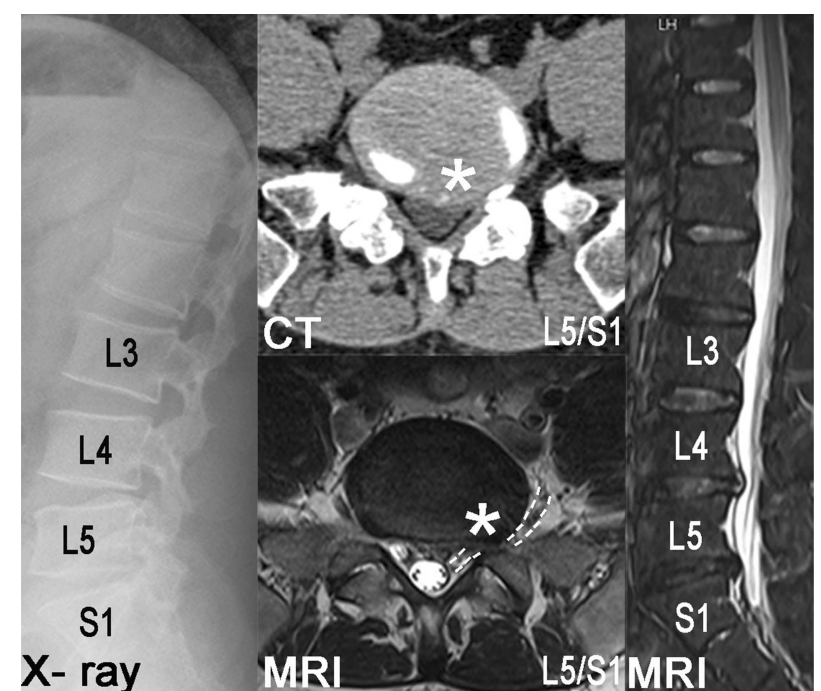

Figure I A case of male patients with chronic low back and leg pain (40 years), axial CT and T2W images (middle), and sagittal T2W images (right) provide clear evidence of discogenic compression (asterisk).

their written informed consent to participate in this study before beginning any of the procedures.

\section{Clinical assessment}

Before rs-fMRI scanning, each participant underwent a battery of tests that including the VAS (0-10) for pain, the Japanese Orthopaedic Association (JOA) Back Pain Evaluation questionnaire ( -6 to 29 ) to examine the impact on neuropathic or nociceptive pain on quality of life, ${ }^{29}$ Fugl-Meyer assessment for sensorimotor impairment measurement and the two-point tactile discrimination (2PD) test to assess tactile spatial resolution ability. ${ }^{30}$

\section{Data acquisition}

All the subjects were scanned using a 3.0 Tesla Trio scanner (Siemens, Munich, Germany) at the First Affiliated Hospital, Nanchang University. During the resting-state fMRI scan, the participants were asked to keep their eyes closed and lie quietly without system thinking in the scanner. The BOLD fMRI scan acquisition included 30 interleaved axial slices with a slice thickness of $4 \mathrm{~mm}$, repetition time $(\mathrm{TR})=2000 \mathrm{~ms}$, echo time $(\mathrm{TE})=30 \mathrm{~ms}$, raw voxel size $=3.44 \times 3.44$, matrix $=64 \times 64$, each functional scan lasted for $8 \mathrm{~min}$. High-resolution T1-weighted images were also acquired using a magnetization-prepared rapid gradient-echo sequence, including 176 sagittal slices with a slice thickness of $1.0 \mathrm{~mm}$, no slice gap, $\mathrm{TR}=1900 \mathrm{~ms}, \mathrm{TE}=2.26 \mathrm{~ms}, \mathrm{FOV}=215 \mathrm{~mm} \times 230 \mathrm{~mm}$, matrix $=240 \times 256$. Additional conventional T2-weighted and T2-fluid-attenuated inversion recovery (FLAIR) sequences were acquired in the lumbar spine and discs from L1 to S3 for a diagnosis of LBLP, another conventional T2-weighted and T2-fluid-attenuated inversion recovery (FLAIR) sequences were acquired in the brain for rule out apparent pathological changes. At the end of the scanning sessions, regarding their waking state in BOLD fMRI, all subjects were required to report that they had not fallen asleep during the scan via the Epworth sleepiness scale questionnaire.

\section{Data preprocessing}

BOLD fMRI preprocessing was performed using the Data Processing and Analysis of Brain Imaging (DPABI) v3.0 (http://www.rfmri.org/dpabi) toolbox, ${ }^{31}$ which is based on the Statistical Parametric Mapping software (SPM12, http://www.fil.ion.ucl.ac.uk/spm/software/spm12/) and running in the MATLAB platform (The MathWorks Inc., Natick, MA, USA). Mainly preprocessing steps included: (1) discarding of the first 10 images to allow for signal equilibration effects; (2) remaining 230 consecutive volumes were corrected for slice-timing and motion corrections (realign); (3) registration of the individual highresolution $\mathrm{T} 1$ images to the mean functional data; (4) transformation and normalization of the resulting realigned functional data to the Montreal Neurological Institute (MNI) standard space with the segmented information from high-resolution T1 template by the Diffeomorphic Anatomical Registration Through Exponentiated Lie Algebra (DARTEL) toolkit; (5) resampling the functional imaging to $3-\mathrm{mm}$ cubic voxels and spatial smoothing of 6-mm FWHM Gaussian kernel; (6) nuisance linear regression with the cerebrospinal fluid, white matter, and Friston-24 head motion parameters (including 6 head motion parameters and their historical effects as well as the 12 corresponding squared items); ${ }^{18}$ and (8) detrend linear drift and temporal band-pass filtered $(0.01-0.1 \mathrm{~Hz})$ to reduce low-frequency drift and high-frequency noise.

Additional, we evaluated the mean absolute displacement of each brain volume to reducing the head motion effects in functional connectivity analysis. The largest mean displacement of all subjects was less than $0.2 \mathrm{~mm}$ according to the criteria of Van Dijk et al, ${ }^{32}$ and there was no significant difference in mean displacement between cLBLP patients and HCs $(0.044 \pm 0.025$ for LBLP and $0.046 \pm 0.039$ for HCs; $P=0.787)$ using a two-sample twotailed $t$-test. 


\section{Temporal filtering and voxel-mirrored homotopic connectivity calculation}

To obtain the interhemispheric homotopic connectivity at the single-voxel level, (1) the normalized mean T1 structural images was used to create a group-specific symmetric T1 template (left-right mirror); (2) individual normalized T1 images were re-normalized to the symmetric T1 template by nonlinear registration; (3) above information of transformation was applied to individual preprocessed functional data, and (4) calculated each pair of symmetrical interhemispheric voxel's time series (Pearson's correlation) as interhemispheric resting-state homotopic connectivity (VMHC). Moreover, this symmetric $\mathrm{T} 1$ template did not include the brain midline $(\mathrm{X}=0)$ area.

In consideration of the frequency property, predefined settings were used to calculate the VMHC, including divided the rs-fMRI data into a typical frequency band $(0.01-0.1 \mathrm{~Hz})$ and five specific frequency bands: slow-6 (0-0.01 Hz), slow-5 (0.01-0.027 Hz), slow-4 (0.027$0.073 \mathrm{~Hz})$, slow-3 $(0.073-0.198 \mathrm{~Hz})$ and slow-2 $(0.198-$ $0.25 \mathrm{~Hz}$ ), according to the Buzsáki framework. ${ }^{33}$

When the VMHC was calculation in each predefined frequency band, then a Fisher's r-to-z transformation were implementing for subsequent group twosample t-tests analyses to determine regional group differences of VMHC data between LBLP and HCs groups (with age and gender treated as covariates; voxel-wise $P<0.01$, Gaussian random field (GRF) theory connected cluster level, $P<0.05$ ). In this study, after defined the region with significant group differences in VMHC, we further evaluated the specific networks influenced by homotopic connectivity (show in Supplementary materials). Then, extract the connectivity coefficient of the regions with significant altered VMHC, we further evaluated the relationships between the altered $\mathrm{VMHC}$ and the clinical metrics (disease duration, VAS, JOA, Fugl-Meyer and 2PD test scores), using partial correlation analyses in SPSS for the LBLP group, with age and gender treated as covariates $(P<0.05$ with Bonferroni corrections).

\section{Discriminant analyses of altered $\mathrm{VMHC}$ values}

We plotted a receiver-operating characteristic (ROC) curve analysis to estimated the optimal cut-off values in the regions with decreased VMHC of cLBLP patients with or without distinguishing ability form HCs. We adopted a leave-one-out cross-validation (LOOCV) test to validate the reproducibility of our results.

\section{Statistical analyses of clinical characteristics}

We compared the demographic and clinical metrics between the two groups using SPSS software (version 18.0; IBM, Armonk, NY, USA). An independent two-sample Student's $t$-test was used to compare the differences in mean head motion, disease duration, VAS, JOA, Fugl-Meyer and 2PD test scores, and the Chi-square test used to compare the differences in gender (categorical data). $P$-values $<0.05$ were considered statistically significant.

\section{Results}

\section{Demographics and clinical characteristics}

In total, 25 cLBLP patients and 27 well-matched HCs were enrolled for the homotopic and heterotopic connectivity analysis in this study, and their clinical characteristics and indices are shown in Table 1. Significantly JOA $(t=14.764, P<0.001)$ and VAS scores $(t=29.569, P<0.001)$ were detected in the cLBLP group than the HCs group. All the cLBLP patients presented with lumbar disk herniation and obvious low-back pain; $15(60 \%)$ and 8 (32\%) patients presented with left and right leg pain and numbness, respective; and $2(8 \%)$ patients presented with bilateral leg symptoms. In those cLBLP patients, 2PD test scores decreased to different degrees in the

Table I Comparison of the demographic data and index of the cLBLP and $\mathrm{HC}$ groups

\begin{tabular}{|l|l|l|l|}
\hline Characteristic & $\begin{array}{l}\text { cLBLP } \\
\text { Patients }\end{array}$ & $\begin{array}{l}\text { Healthy } \\
\text { controls }\end{array}$ & \multirow{2}{*}{-values } \\
\cline { 2 - 3 } & Mean (SD) & Mean (SD) & \\
\hline Gender (M/F) & $10 / 15$ & $15 / \mathrm{I} 2$ & $0.283^{\#}$ \\
Age (y) & $55.56(8.67)$ & $53.37(8.18)$ & 0.354 \\
Duration of symptoms & $38.96(50.26)$ & $\mathrm{n} / \mathrm{a}(\mathrm{n} / \mathrm{a})$ & $\mathrm{n} / \mathrm{a}$ \\
(months) & & & \\
VAS scores & $5.84(\mathrm{I} .0 \mathrm{I})$ & $28.92(0.27)$ & $<0.000 \mathrm{I}$ \\
JOA scores & $14.16(5.13)$ & $28.92(0.27)$ & $<0.000 \mathrm{I}$ \\
Fugl-Meyer scores & $20.12(\mathrm{I} .98)$ & $\mathrm{n} / \mathrm{a}(\mathrm{n} / \mathrm{a})$ & $\mathrm{n} / \mathrm{a}$ \\
ICV (mm $\left.{ }^{3}\right)$ & $0.775(0.028)$ & $0.782(0.018)$ & $<0.35 \mathrm{I}$ \\
Mean head motion* & $0.044(0.025)$ & $0.046(0.039)$ & 0.787 \\
\hline
\end{tabular}

Note: CLBLP, chronic low-back and leg pain; F, female; ICV, intracranial volume; JOA, Japanese Orthopaedic Association; M, male; n/a, not available; SD, standard deviation; VAS, visual analog scale; $y$, years. ${ }^{~}$ Chi-square tests; *Head motions were evaluated according to the framewise displacement (FD) criteria described by Van Dijk et al (2012). ${ }^{32}$ 
right $(30.12 \pm 1.37 \mathrm{~mm})$ and left $(30.04 \pm 1.23 \mathrm{~mm})$ feet and the right $(24.9 \pm 1.18 \mathrm{~mm})$ and left $(25.0 \pm 1.22 \mathrm{~mm})$ hands. The cLBLP and HCs group exhibited no significant differences in age $(P=0.354)$, or sex $(P=0.283)$, mean framewise displacement $(P=0.787)$ or intracranial volume $(P=0.351)$.

\section{Homotopic connectivity pattern and group comparisons within the frequency subbands}

In the typical frequency band $(0.01-0.1 \mathrm{~Hz})$, homotopic connectivity spatial distributions shown in Figure 2A; while the cLBLP patients showed only reduce $\mathrm{VMHC}$ in the right and left inferior temporal gyrus (ITG), the right and left basal ganglia (BG), the right and left middle frontal gyrus (MFG), the right and left superior temporal gyrus (STG), the right and left medial prefrontal cortex (mPFC) $(P<0.01$, GRF-correction with cluster level of $P<0.05$; Figure 2B and Table 2).

In five specific frequency bands, similar spatial distributions of VMHC was detected in the cLBLP and HCs group, but relatively strongly connected with lower frequency bands (shown in Figure S1). Figure 3 and Table 3 showed the alterations of VMHC in the five specific frequency bands using voxel-based analyses in the LBLP patients and HCs $(P<0.01, \mathrm{GRF}$-correction with cluster level of $P<0.05)$.

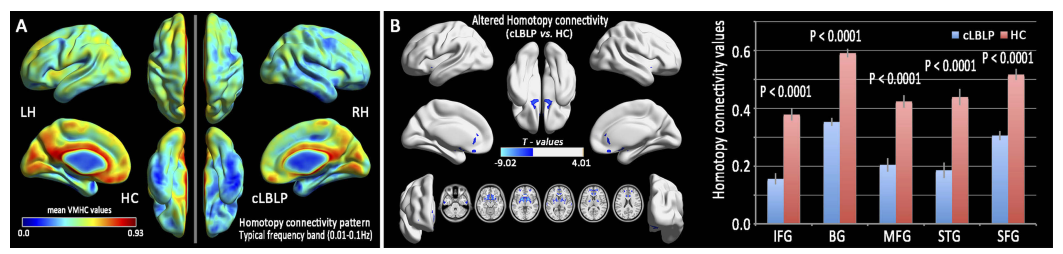

Figure 2 Group means (A) and group comparison (B) of homotopy connectivity in the CLBLP and HC groups in the typical (0.0I-0.I Hz) frequency bands. Note: Column B shows the group comparison at voxels level (left, $P<0.0$, GRF-corrected at a cluster level of $P<0.05$ ) and cluster level (right), while details information can be found in Table 2 .

Table 2 Group comparison of homotopic connectivity between the cLBLP patient and HC subject in the typical (0.0I-0.I Hz) frequency bands $(P<0.01$, GRF-corrected for multiple comparisons at a cluster level of $P<0.05)$

\begin{tabular}{|l|l|l|l|l|l|l|l|}
\hline \multirow{2}{*}{ Brain region } & \multirow{2}{*}{ BA } & \multirow{2}{*}{ Voxels size } & \multirow{2}{*}{ Peak t-value } & \multicolumn{2}{|l|}{ Peak coordinates in MNI space } & \multirow{2}{*}{ Effect size (Cohen's d) } \\
\cline { 5 - 7 } & & & & $\mathbf{X}$ & $\mathbf{Y}$ & $\mathbf{Z}$ & \\
\hline Inferior temporal gyrus (ITG) & 20 & 29 & -5.432 & \pm 54 & -15 & -36 & 2.136 \\
Basal ganglia (BG) & & 537 & -9.019 & \pm 9 & 12 & -9 & 3.180 \\
Middle frontal gyrus (MFG) & 10 & 26 & -4.999 & \pm 30 & 54 & -3 & 1.870 \\
Superior temporal gyrus (STG) & 22 & 20 & -5.434 & \pm 55 & -24 & 0 & 0.657 \\
Medial prefrontal cortex (mPFC) & 10 & 129 & -6.256 & \pm 18 & 57 & 9 & 0.736 \\
\hline
\end{tabular}

Note: BA: Brodmann's area. t: statistical value of peak voxels showing significant differences between the two groups (negative values: Clblp < HC; positive values: cLBLP > HC). MNI: Montreal Neurological Institute Coordinate System; X, Y, Z: coordinates of primary peak locations in the MNI space (same as all figure and table).

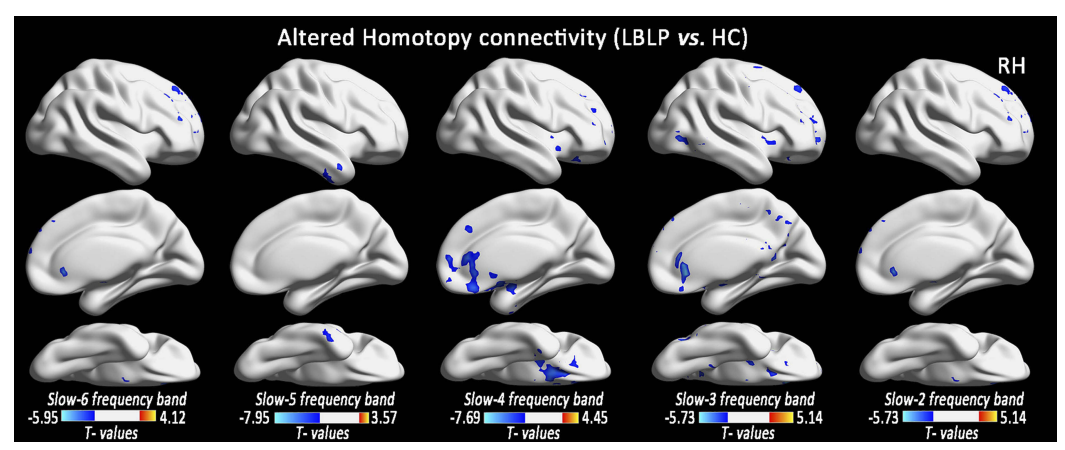

Figure 3 Alterations of VMHC between the cLBLP patients and HCs in the five specific (slow- 6 to slow- 2 ) frequency bands (two-tailed, voxel-level $P<0.01$, GRF correction, cluster-level $P<0.05)$. 
Table 3 Alterations of VMHC of five specific frequency bands between the CLBLP and HC group (two-tailed, voxel-level $P<0.01$, GRF correction, cluster-level $P<0.05$ )

\begin{tabular}{|c|c|c|c|c|c|c|c|}
\hline \multirow[t]{2}{*}{ Brain regions } & \multirow[t]{2}{*}{ BA } & \multirow[t]{2}{*}{ Peak T-scores } & \multicolumn{3}{|c|}{ MNI coordinates } & \multirow{2}{*}{$\begin{array}{l}\text { Cluster size } \\
\text { (voxels) }\end{array}$} & \multirow{2}{*}{$\begin{array}{l}\text { Effect size } \\
\text { (Cohen's d) }\end{array}$} \\
\hline & & & $\mathbf{x}$ & $y$ & $\mathbf{z}$ & & \\
\hline \multicolumn{8}{|c|}{ Altered VMHC at slow-6 (0-0.0I Hz) band (cLBLP vs HC) } \\
\hline Medial frontal gyrus/anterior cingulate cortex & 25,47 & -5.590 & \pm 15 & 21 & -18 & 803 & \\
\hline Inferior frontal gyrus & 38,44 & -5.147 & \pm 48 & 36 & -6 & 126 & \\
\hline Superior temporal gyrus & 22,42 & -3.952 & \pm 60 & -33 & -6 & 94 & \\
\hline Precuneus & 7,31 & -4.864 & \pm 3 & -57 & 27 & 101 & \\
\hline \multicolumn{8}{|c|}{ Altered VMHC at slow-5 $(0.0 \mathrm{I}-0.027 \mathrm{~Hz})$ band (cLBLP vs $\mathrm{HC})$} \\
\hline Middle temporal gyrus & 20,21 & -4.426 & \pm 60 & -6 & -33 & 174 & \\
\hline Superior temporal gyrus/putamen & 47,22 & -7.954 & \pm 15 & 6 & -6 & 2137 & \\
\hline \multicolumn{8}{|c|}{ Altered VMHC at slow-4 $(0.027-0.073 \mathrm{~Hz})$ band (cLBLP vs $\mathrm{HC})$} \\
\hline Medial and superior frontal gyrus/basal ganglia & 10,47 & -7.688 & \pm 9 & 12 & -9 & 1802 & \\
\hline \multicolumn{8}{|c|}{ Altered VMHC at slow-3 $(0.073-0.167 \mathrm{~Hz})$ band (cLBLP vs HC) } \\
\hline Middle temporal gyrus & 21,37 & -4.660 & \pm 42 & -75 & 0 & 168 & \\
\hline Putamen & & -5.714 & \pm 12 & 15 & -6 & 875 & \\
\hline Superior frontal gyrus & 9,10 & -4.970 & \pm 18 & 51 & 10 & 437 & \\
\hline Precuneus & 7 & -5.525 & \pm 3 & -48 & 51 & 269 & \\
\hline Precentral and postcentral cortex & 6,3 & -3.991 & \pm 33 & -12 & 51 & 261 & \\
\hline \multicolumn{8}{|c|}{ Altered VMHC at slow-2 $(0.167-0.25 \mathrm{~Hz})$ band (cLBLP vs $\mathrm{HC})$} \\
\hline Thalamus/basal ganglia & & -5.621 & \pm 18 & 18 & 0 & 238 & \\
\hline Superior frontal gyrus & 10 & -4.590 & \pm 42 & 45 & 18 & 163 & \\
\hline Middle Frontal Gyrus & $10 \square 46$ & -5.246 & \pm 15 & 42 & 51 & 169 & \\
\hline
\end{tabular}

Interactions between disease status and the five specific frequency bands

The full-factorial analysis showed significant interactions between disease status and the five specific frequency bands of the cerebellum anterior lobe (CAL), the middle temporal gyrus (MTG) and the anterior cingulate cortex (ACC) (Figure 4 and Table 4).

\section{Discriminatory analysis of the homotopic connectivity coefficient}

To verify the ability to distinguish the cLBLP patients from the HCs, the mean connectivity coefficients of reducing VMHC in the typical frequency band $(0.01-0.1 \mathrm{~Hz})$ were extracted from the cLBLP and HCs group. The ROC analysis revealed that the areas under the curves (AUCs) were 0.936 for right and left ITG, 0.996 for the right and left BG, 0.916 for the right and left MFG, 0.889 for the right and left STG, and 0.944 for the right and left mPFC (Figure 5 and Table 5). The LOOCV showed cross-validated AUCs to be
$0.936 \pm 0.004(0.934-0.949)$ for the right or left ITG, 0.995 \pm 0.008 (0.995-0.997) for the right or left BG, $0.915 \pm 0.006$ (0.912-0.948) for the right or left MFG, $0.889 \pm 0.006$ (0.884-0.908) for the right or left STG, and $0.944 \pm 0.005$ (0.941-0.977) for the right or left mPFC. In the five specific frequency bands, the disrupted VMHC regions also showed a greater ability to distinguish the disease from $\mathrm{HCs}$ (Figure 5 and Table 6).

\section{Clinical metrics associated with homotopic connectivity coefficient in cLBLP patients}

In typical frequency band $(0.01-0.1 \mathrm{~Hz})$, significant correlations were observed between the VMHC values of BG and the values of $2 \mathrm{PD}$ of right hand $(\rho=-0.446, P=0.033$ ), between the VMHC values of $\mathrm{mPFC}$ and the VAS score ( $\rho=0.476, P=0.022$ ) (Figure 6) in the cLBLP group; while no significant relationship was observed between the altered VMHC and the clinical metrics, such as the disease 


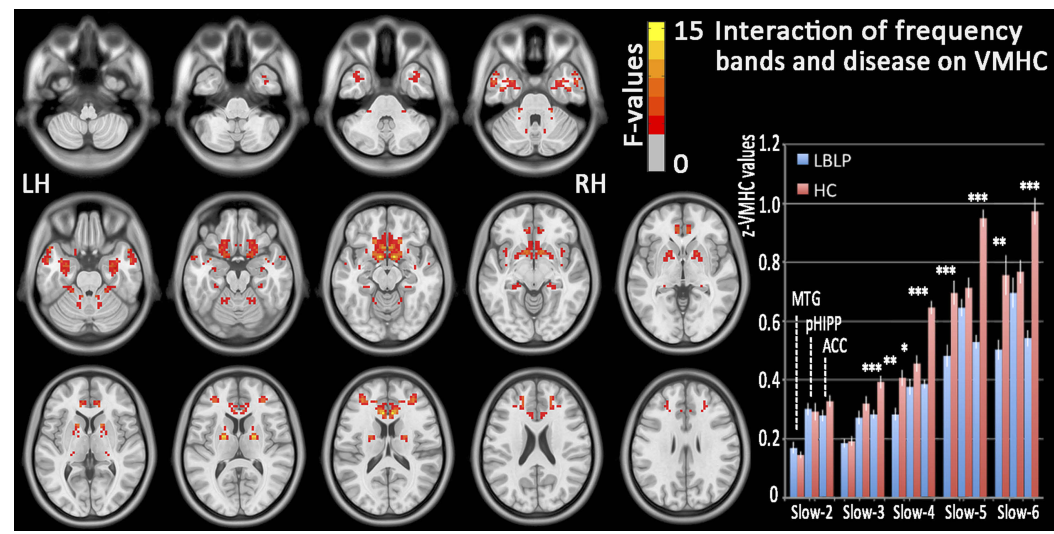

Figure 4 Interactions between the five specific frequency bands (slow-2 to slow-6) and group (cLBLP patients and HCs) on VMHC at voxels level (left) and cluster level (right).

Table 4 Significant interaction between disease status and the five specific frequency bands on VMHC (full-factorial design, $2 \times 5$ )

\begin{tabular}{|l|l|l|l|l|l|l|}
\hline Brain regions & \multirow{2}{*}{ BA } & Peak F-scores & \multicolumn{2}{l|}{ MNI coordinates } & Cluster size (voxels) \\
\cline { 4 - 7 } & & & $\mathbf{x}$ & $\mathbf{y}$ & $\mathbf{z}$ & \\
\hline Middle temporal gyrus & 21,38 & 9.646 & \pm 51 & 15 & -30 & 211 \\
Cerebellum anterior lobe & & 7.882 & \pm 21 & -33 & -6 & 105 \\
Anterior cingulate cortex & 25,47 & 15.681 & \pm 6 & 30 & 12 & 525 \\
\hline
\end{tabular}

Note: All clusters were analyzed using a two-tailed test with a voxel-level threshold of $P<0.0 \mathrm{I}$, GRF correction and cluster-level of $P<0.05$.

duration, and JOA Back Pain Evaluation scores $(P=0.061-$ 0.991).

Similarly, significant correlations were observed between the VMHC values of slow-4 band of medial and superior frontal gyrus/basal ganglia and the values of 2 PD of right hand ( $\rho=-0.624, P=0.0015)$, between the VMHC values of slow-2 band of thalamus/basal ganglia and the values of $2 \mathrm{PD}$ of right hand ( $\rho=-0.470, P=0.024$ ), between the VMHC values of slow-2 band of superior frontal gyrus and the values of 2PD of right hand $(\rho=$ $-0.456, P=0.029$ ).

\section{Discussion}

We investigated the interhemispheric functional coordination in patients with cLBLP. We showed disease related to disrupting interhemispheric connectivity of cLBLP patients, which was summarized as (1) a significantly decreased VMHC in the typical and then five specific frequency bands; (2) further demonstrated significant interactions between disease status and the five specific frequency bands of several regions; (3) significant correlation was observed between the decreased VMHC values and VAS score, 2PD test score in the cLBLP patients.

\section{Disrupted interhemispheric connectivity in cLBLP patients in typical frequency bands}

The correlation between the fMRI signals and the mirrored brain regions at rest has been developed to estimate the strength of resting-state functional connectivity, VMHC, as one of the reliable and reproducible metrics of the brain intrinsic architecture. ${ }^{16,18}$ In this study, we observed only significantly reduced homotopic connectivity in cLBLP patients in the ITG, the BG, the MFG, the STG, and the mPFC.

ITG mainly connected with left and right MFG, contralateral ITG, while evidence of functional neuroimaging studies has revealed that ITG has a role in identifying objects and/or processes the visual information processing, memory, and spatial awareness. ${ }^{34}$ Some studies have linked the ITG to represents stored limbs-postures for planning and predicting object-directed actions and activities. $^{35}$ In this study, the mainly influenced specific (heterotopic connectivity) network of decreased ITG is contralateral ITG, right and left MFG. The strategic location and the effect of connections suggest that ITG is a major association area that subserves readiness or contingency awareness of limb in patients with cLBLP. 

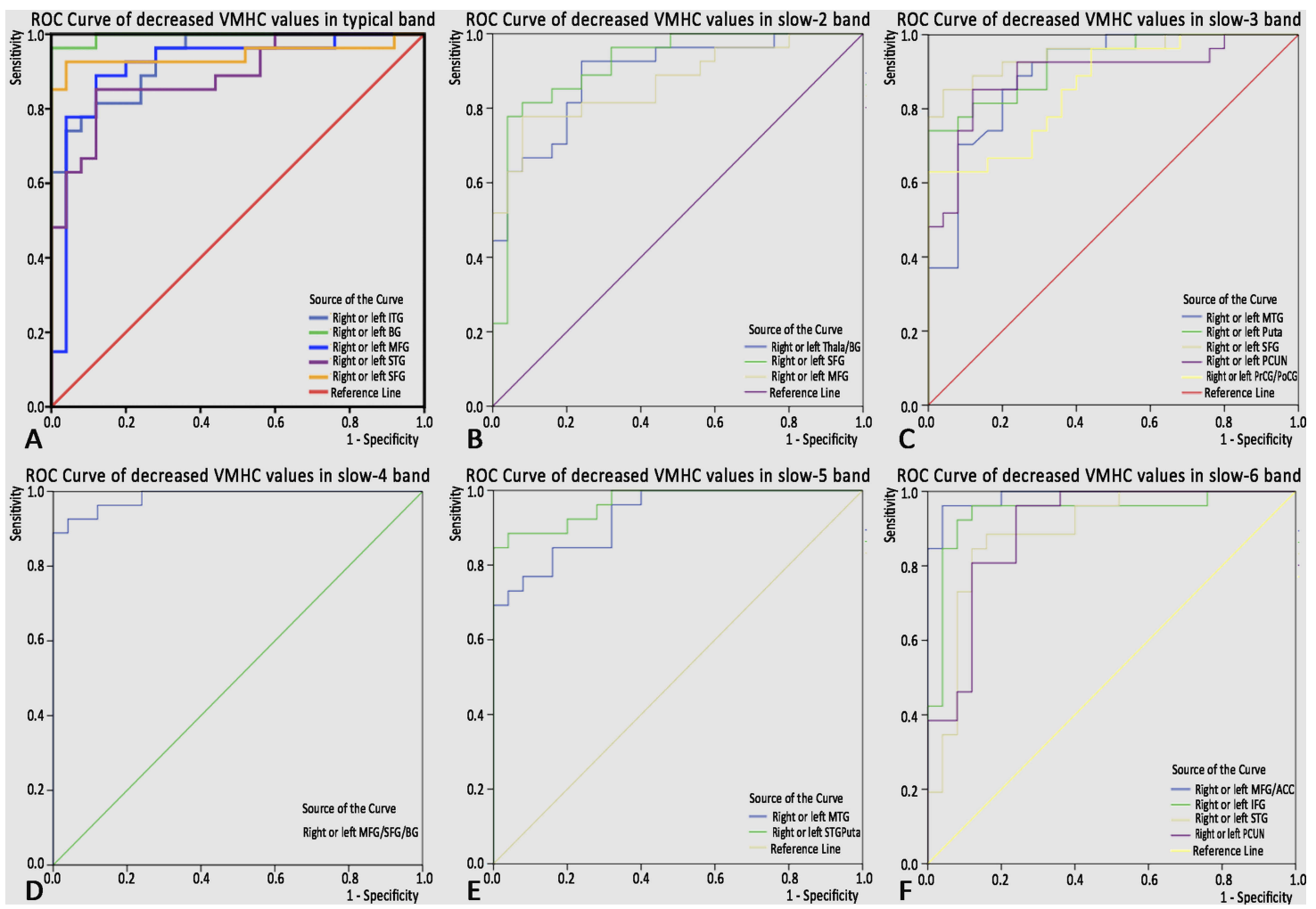

Figure 5 Significantly decreased VMHC values in the ROC analysis showing a moderate differentiating ability for cLBLP.

Table 5 ROC discriminatory analysis of the homotopic connectivity coefficient in the typical $(0.0 \mathrm{I}-0 . \mathrm{I} \mathrm{Hz})$ frequency bands

\begin{tabular}{|l|l|l|l|l|}
\hline Brain regions & Area Under the Curve & Cut-off point & Sensitivity & Specificity \\
\hline Inferior temporal gyrus & 0.936 & $0.298^{\mathrm{a}}$ & $81.5 \%(22 / 27)$ & $92.0 \%(23 / 25)$ \\
Basal ganglia & 0.996 & 0.475 & $96.3 \%(26 / 27)$ & $100 \%(25 / 25)$ \\
Middle frontal gyrus & 0.916 & 0.302 & $88.9 \%(24 / 27)$ & $88.0 \%(22 / 25)$ \\
Superior temporal gyrus & 0.889 & 0.288 & $85.2 \%(23 / 27)$ & $88.0 \%(22 / 25)$ \\
Superior frontal gyrus & 0.944 & 0.426 & $92.6 \%(25 / 27)$ & $96.0 \%(22 / 25)$ \\
\hline
\end{tabular}

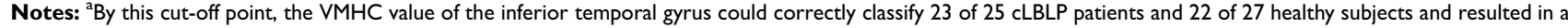
specificity of $92.0 \%$ and a sensitivity of $81.5 \%$. The means of other cut-off points were similar.

Another region with decreased homotopic connectivity, BG mainly influenced bilateral anterior cingulate cortex/MFG in this study. As same as, this study observed that decreased VMHC values of BG negative with the values of 2PD of the right hand in patients with cLBLP. 2PD is commonly used for assessing tactile spatial discrimination ability with highly intra-observer reliability. ${ }^{36}$ Decreased VMHC values of BG meaning interhemispheric incoordination is influenced to tactile discrimination ability. But corresponding to the function of BG, we know, it are not simply involved in the control of movement, but more related to aspects of cognitive function. ${ }^{36}$ Decreased heterotopic connectivity between the $\mathrm{BG}$ and anterior cingulate cortex/MFG hinting an impairment in certain higher-level functions, such as attention allocation, contingency awareness, and optimal decision making. It might originate from longterm, chronic pain, and numbness in cLBLP patients.

When decreased VMHC of MFG and MPFC were observed, evidence from disrupting those regions connected with the default-mode and attention network by heterotopic connectivity analyses. In other words, the MFG and mPFC are an important part of the defaultmode and attention network. Studying with fMRI has shown an abnormal response to the brain default mode network of an attention task, even disrupts normal activity during the brain resting state in chronic back pain patients. ${ }^{7,37}$ It is known that an intrinsic activity 
Table 6 ROC discriminatory analysis of the homotopic connectivity coefficient in five specific frequency bands

\begin{tabular}{|c|c|c|c|c|}
\hline Brain regions & Area Under the Curve & Cut-off point & Sensitivity & Specificity \\
\hline \multicolumn{5}{|c|}{ The regions with altered VMHC at slow- $6(0-0.01 \mathrm{~Hz})$ band (cLBLP vs HC) } \\
\hline Medial frontal gyrus/anterior cingulate cortex & 0.988 & 0.430 & $96.2 \%(26 / 27)$ & $96.0 \%(24 / 25)$ \\
\hline Inferior frontal gyrus & 0.943 & 0.344 & $92.6 \%(25 / 27)$ & $92.0 \%(23 / 25)$ \\
\hline Superior temporal gyrus & 0.892 & 0.453 & $84.6 \%(23 / 27)$ & $88.0 \%(22 / 25)$ \\
\hline Precuneus & 0.902 & 0.617 & $96.2 \%(26 / 27)$ & $76.0 \%(19 / 25)$ \\
\hline \multicolumn{5}{|c|}{ The regions with altered VMHC at slow-5 $(0.01-0.027 \mathrm{~Hz})$ band (cLBLP vs HC) } \\
\hline Middle temporal gyrus & 0.931 & 0.471 & $77.8 \%(20 / 27)$ & $96.0 \%(24 / 25)$ \\
\hline Superior temporal gyrus/putamen & 0.968 & 0.479 & $88.9 \%(24 / 27)$ & $100 \%(25 / 25)$ \\
\hline \multicolumn{5}{|c|}{ The regions with altered VMHC at slow- $4(0.027-0.073 \mathrm{~Hz})$ band (cLBLP vs HC) } \\
\hline Medial and superior frontal gyrus/basal ganglia & 0.985 & 0.419 & $92.6 \%(25 / 27)$ & $96.0 \%(24 / 25)$ \\
\hline \multicolumn{5}{|c|}{ The regions with altered VMHC at slow-3 $(0.073-0.167 \mathrm{~Hz})$ band (cLBLP vs HC) } \\
\hline Middle temporal gyrus & 0.897 & 0.232 & $85.2 \%(23 / 27)$ & $80.0 \%(20 / 25)$ \\
\hline Putamen & 0.927 & 0.354 & $74.1 \%(20 / 27)$ & $100 \%(25 / 25)$ \\
\hline Superior frontal gyrus & 0.950 & 0.273 & $85.2 \%(23 / 27)$ & $92.0 \%(23 / 25)$ \\
\hline Precuneus & 0.892 & 0.477 & $85.2 \%(23 / 27)$ & $88.0 \%(22 / 25)$ \\
\hline Precentral and postcentral cortex & 0.862 & 0.279 & $63 \%(17 / 27)$ & $100 \%(25 / 25)$ \\
\hline \multicolumn{5}{|c|}{ The regions with altered VMHC at slow-2 $(0.167-0.25 \mathrm{~Hz})$ band (cLBLP vs HC) } \\
\hline Thalamus/basal ganglia & 0.890 & 0.346 & $92.6 \%(25 / 27)$ & $76.0 \%(19 / 25)$ \\
\hline Superior frontal gyrus & 0.919 & 0.198 & $85.2 \%(23 / 27)$ & $84.0 \%(21 / 25)$ \\
\hline Middle Frontal gyrus & 0.870 & 0.396 & $77.8 \%(2 \mathrm{I} / 27)$ & $92.0 \%(23 / 25)$ \\
\hline
\end{tabular}
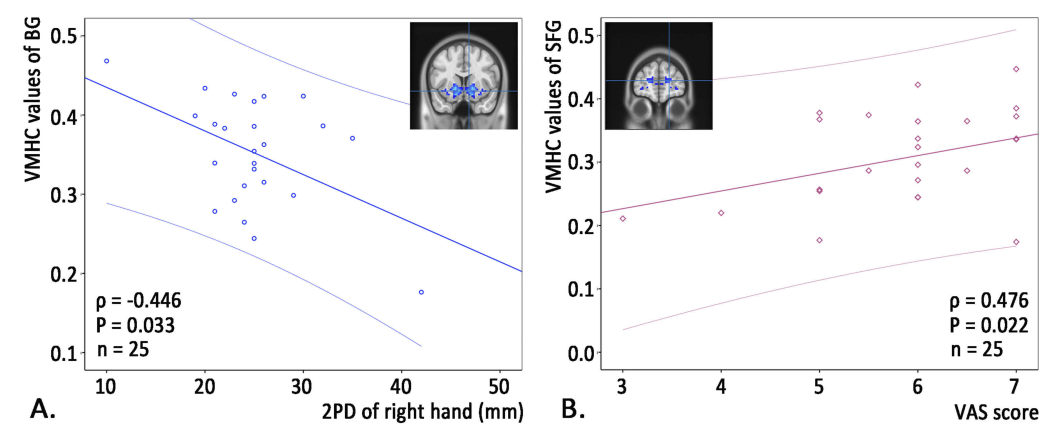

Figure 6 Correlations between the VMHC coefficients and the clinical index in the cLBLP patients in the typical $(0.0 \mathrm{I}-0.1 \mathrm{~Hz})$ frequency bands.

in the default mode network when the absence of explicit brain input or output or the brain is at wakeful rest. But for cLBLP patients, the pain and numbness become a spontaneous percept, this status might result in a different "intrinsic" organization. In this study, we found a VAS score positively related alterations in the homotopic connectivity of the mPFC, together with others in this study about disrupted homotopic and heterotopic connectivity (show in Figure S2) indicated that chronic stimulation of extrinsic participated in the central functional reorganization of the default mode network. In short, this new observation in VMHC is consistent with previous intrinsic functional connectivity showing that due to a mismatch of the temporal patterns of neuronal intrinsic activity or of the functional statuses, which might play a critical role in attention, memory, prospection, and self-processing in cLBLP patients. 


\section{Interactions between frequency-based VMHC and disease status in cLBLP patients}

Significant interactions were observed in several regions, involving unconscious information processing (CAL), contemplating distance processing (MTG), and certain higher-level functions (ACC), between frequency-based VMHC and disease status in cLBLP patients. Inputs into the CAL are mainly from the spinal cord and responsible for mediating unconscious proprioception. A recent study has shown speed-specific neuro-functional and -structural correlates with the $\mathrm{CAL},{ }^{38}$ and it can deteriorate in the alcoholics associated with sensorimotor integration. ${ }^{39}$ The exact function of MTG is unknown, but it has been connected with processes as different as contemplating distance, and multimodal semantic processing. ${ }^{40}$ While the ACC is involved monitoring the experience of pain and emotional reaction. Regarding the intrinsic activity, distinct frequency-specific functional properties may arise from the various cytoarchitectural or synaptic types in this area. ${ }^{17,41}$ In the above regions, more frequency-dependent properties or increased richness in scales of VMHC measures, suggesting that different frequency bands may have specific pathological relevance to this region, might explain the clinical symptom.

In this study, different altered pattern of VMHC in the five specific frequency bands, and it influenced heterotopic connectivity (shown in Figures S3 and S4), implied an interhemispheric interaction of pain and paresthesia (numbness) in the frequency-related alterations in specific brain regions in the patients with LBLP. Baliki et al ${ }^{25}$ found that a closed relationship exists anatomical structures and the intrinsic activity in a specific frequency bands. Our findings may underlie the neurophysiological basis of the interhemispheric functional coordination. These findings suggest that specific frequency ranges, even high-frequency bands, should be selected to detect pain-related homotopic connectivity in future studies of cLBLP patients.

\section{Discriminant validity of altered $\mathrm{VMHC}$ values in CLBLP patients}

Currently, the clinical diagnosis of cLBLP is base on physical examination and the evidence of discogenic compression on a lumbar CT and/or MRI. Therefore, an accurate and objective diagnosis of central sensitization or central plasticity in cLBLP has high clinical value to explore the new treatment or prognostic evaluation. With this aim, we showed that disrupted VMHC differentiated the plasticity brain of cLBLP from healthy individuals with high sensitivity and specificity, using a ROC analysis. To the best of our knowledge, few studies have attempted to use intrinsic functional metrics as a classification tool for judge central sensitization in chronic pain patients. Hence, this discriminant analyses presented here, as a preferential candidate, are preliminary because they have not been verified in a separate group. It needs further studies are warranted in the future.

\section{Limitations}

This exploratory finding is promising, but several issues need to be noted. First, to expose the intrinsic functional architecture stabile and precisely is challenging in a relatively small sample size. Thus, the interesting findings will require replication to increase the statistical power in a larger sample size study. Second, the human brain is unsymmetrical. We tried to use symmetrical standard template to improve the functional coherence in interhemispheric homotopic regions. Hence, morphometric asymmetry could not account for the altered VMHC. Third, these current results were obtained under pain and numbness of limb condition. What is also unclear is the mechanism for leg pain with low back pain afferent input on central neurons. Future studies should combine both pain and pain-free condition, or other task-based fMRI to observe interhemispheric connectivity in cLBLP patients. Additionally, the altered connection strength of this study without characterizing the anatomical basis of the interhemispheric connection (e.g. diffusion tensor tractography), the underlying mechanisms of disrupted VMHC in cLBLP, and the difference in intrinsic functional connectivity with other chronic low back pain. It needs further studies to expose to future research.

\section{Conclusion}

In conclusion, the present study found that cLBLP patients showed disrupted interhemispheric coordination, with different heterotopic connectivity patterns in these regions with decreased VMHC. Moreover, VAS scores correlated alteration of VMHC may provide a useful and sensitive screening approach for observed interhemispheric "intrinsic" organization in patients with chronic pain.

\section{Acknowledgments}

The authors would like to thank all of the participants in this study. This study was supported by the National 
Natural Science Foundation of China (81560284 and 81771808), the Key Science and Technology Financing Projects of the Jiangxi Provincial Education Department (GJJ170003), and the Distinguished Young Scholars of Jiangxi Province (20171BCB23089). The funders had no role in the study design, data collection, and analysis, decision to publish, or preparation of the manuscript.

\section{Author contributions}

All authors contributed to data analysis, drafting or revising the article, gave final approval of the version to be published, and agree to be accountable for all aspects of the work.

\section{Disclosure}

The authors report no conflicts of interest in this work. None of the authors have any personal or financial involvement with organizations that have a financial interest in its content.

\section{References}

1. Zhang YG, Guo TM, Xiong G, Wu SX. Clinical diagnosis for discogenic low back pain. Int J Biol Sci. 2009;5(7):647-658. doi:10.7150/ijbs.5.647

2. Stynes S, Konstantinou K, Dunn KM. Classification of patients with low back-related leg pain: a systematic review. BMC Musculoskelet Disord. 2016;17:226. doi:10.1186/s12891-016-1134-4

3. Konstantinou K, Hider SL, Jordan JL, Lewis M, Dunn KM, Hay EM. The impact of low back-related leg pain on outcomes as compared with low back pain alone: a systematic review of the literature. Clin J Pain. 2013;29(7):644-654. doi:10.1097/AJP.0b013e31826f9a52

4. Konstantinou K, Dunn KM, Ogollah R, Vogel S, Hay EM. ATLAS study research team. Characteristics of patients with low back and leg pain seeking treatment in primary care: baseline results from the ATLAS cohort study. BMC Musculoskelet Disord. 2015;16:332.

5. Ebrahimi S, Kamali F, Razeghi M, Haghpanah SA. Comparison of the trunk-pelvis and lower extremities sagittal plane inter-segmental coordination and variability during walking in persons with and without chronic low back pain. Hum Mov Sci. 2017;52:55-66. doi:10.1016/j.humov.2017.01.004

6. Anukoolkarn K, Vongsirinavarat M, Bovonsunthonchai S, Vachalathiti R. Plantar pressure distribution pattern during mid-stance phase of the gait in patients with chronic non-specific low back pain. J Med Assoc Thai. 2015;98(9):896-901.

7. Zhou F, Gu L, Hong S, et al. Altered low-frequency oscillation amplitude of resting state-fMRI in patients with discogenic low-back and leg pain. J Pain Res. 2018;11:165-176. doi:10.2147/JPR.S151562

8. Zhou F, Wu L, Guo L, Zhang Y, Zeng X. Local connectivity of the resting brain connectome in patients with low back-related leg pain: a multiscale frequency-related Kendall's coefficient of concordance and coherence-regional homogeneity study. Neuroimage Clin. 2019;21:101661. doi:10.1016/j.nicl.2019.101661

9. Sharma NK, McCarson K, Van Dillen L, Lentz A, Khan T, Cirstea CM. Primary somatosensory cortex in chronic low back pain - a HMRS study. J Pain Res. 2011;4:143-150. doi:10.2147/JPR.S19297

10. Fritz HC, McAuley JH, Wittfeld K, et al. Chronic back pain is associated with decreased prefrontal and anterior insular gray matter: results from a population-based cohort study. J Pain. 2016;17 (1):111-118. doi:10.1016/j.jpain.2015.10.003
11. Cauda F, Palermo S, Costa T, et al. Gray matter alterations in chronic pain: a network-oriented meta-analytic approach. Neuroimage Clin. 2014;4:676-686. doi:10.1016/j.nicl.2014.04.007

12. Dolman AJ, Loggia ML, Edwards RR, et al. Phenotype matters: the absence of a positive association between cortical thinning and chronic low back pain when controlling for salient clinical variables. Clin J Pain. 2014;30(10):839-845. doi:10.1097/AJP.0000000000000043

13. Ung H, Brown JE, Johnson KA, Younger J, Hush J, Mackey S. Multivariate classification of structural MRI data detects chronic low back pain. Cereb Cortex. 2014;24(4):1037-1044. doi:10.1093/ cercor/bhs 378

14. Buckalew N, Haut MW, Aizenstein H, et al. Differences in brain structure and function in older adults with self-reported disabling and nondisabling chronic low back pain. Pain Med. 2010;11(8):11831197. doi:10.1111/j.1526-4637.2010.00899.x

15. Shi $\mathrm{Y}$, Zong $\mathrm{M}, \mathrm{Xu} \mathrm{X}$, et al. Diffusion tensor imaging with quantitative evaluation and fiber tractography of lumbar nerve roots in sciatica. Eur $J$ Radiol. 2015;84(4):690-695. doi:10.1016/j.ejrad.2015.01.006

16. Zuo XN, Kelly C, Di Martino A, et al. Growing together and growing apart: regional and sex differences in the lifespan developmental trajectories of functional homotopy. $J$ Neurosci. 2010;30 (45):15034-15043. doi:10.1523/JNEUROSCI.2612-10.2010

17. Gee DG, Biswal BB, Kelly C, et al. Low frequency fluctuations reveal integrated and segregated processing among the cerebral hemispheres. Neuroimage. 2011;54(1):517-527. doi:10.1016/j. neuroimage.2010.05.073

18. Yan CG, Craddock RC, Zuo XN, Zang YF, Milham MP. Standardizing the intrinsic brain: towards robust measurement of inter-individual variation in 1000 functional connectomes. Neuroimage. 2013;80:246262. doi:10.1016/j.neuroimage.2013.04.081

19. Sevel LS, Letzen JE, Staud R, Robinson ME. Interhemispheric dorsolateral prefrontal cortex connectivity is associated with individual differences in pain sensitivity in healthy controls. Brain Connect. 2016;6(5):357-364. doi:10.1089/brain.2015.0405

20. Di Pietro F, Stanton TR, Moseley GL, Lotze M, McAuley JH. Interhemispheric somatosensory differences in chronic pain reflect abnormality of the healthy side. Hum Brain Mapp. 2015;36(2):508518. doi:10.1002/hbm. 22643

21. Morishita T, Inoue T. Brain stimulation therapy for central poststroke pain from a perspective of interhemispheric neural network remodeling. Front Hum Neurosci. 2016;10:166. doi:10.3389/fnhum. 2016.00166

22. Zhou F, Huang S, Zhuang Y, Gao L, Gong H. Frequency-dependent changes in local intrinsic oscillations in chronic primary insomnia: a study of the amplitude of low-frequency fluctuations in the resting state. Neuroimage Clin. 2017;15:458-465. doi:10.1016/j.nicl.2016.05.011

23. Buzsaki G, Draguhn A. Neuronal oscillations in cortical networks. Science. 2004;304(5679):1926-1929. doi:10.1126/science.1099745

24. Zuo XN, Di Martino A, Kelly C, et al. The oscillating brain: complex and reliable. NeuroImage. 2010;49(2):1432-1445. doi:10.1016/j. neuroimage.2009.09.037

25. Baliki MN, Baria AT, Apkarian AV. The cortical rhythms of chronic back pain. J Neurosci. 2011;31(39):13981-13990. doi:10.1523/ JNEUROSCI.1984-11.2011

26. Iwata M, Leblanc BW, Kadasi LM, Zerah ML, Cosgrove RG, Saab CY. High-frequency stimulation in the ventral posterolateral thalamus reverses electrophysiologic changes and hyperalgesia in a rat model of peripheral neuropathic pain. Pain. 2011;152(11):2505-2513. doi:10.1016/j.pain.2011.07.011

27. Leblanc BW, Lii TR, Silverman AE, Alleyne RT, Saab CY. Cortical theta is increased while thalamocortical coherence is decreased in rat models of acute and chronic pain. Pain. 2014;155(4):773-782. doi:10.1016/j.pain.2014.01.013

28. Malik KM, Cohen SP, Walega DR, Benzon HT. Diagnostic criteria and treatment of discogenic pain: a systematic review of recent clinical literature. Spine. 2013;13(11):1675-1689. doi:10.1016/j.spinee.2013.06.063 
29. Yonenobu K, Abumi K, Nagata K, Taketomi E, Ueyama K. Interobserver and intraobserver reliability of the Japanese Orthopaedic Association scoring system for evaluation of cervical compression myelopathy. Spine. 2001;26(17):1890-1894. doi:10.10 97/00007632-200109010-00014

30. Boldt R, Gogulski J, Gúzmanlopéz J, Carlson S, Pertovaara A. Twopoint tactile discrimination ability is influenced by temporal features of stimulation. Exp Brain Res. 2014;232(7):2179-2185. doi:10.1007/ s00221-014-3908-y

31. Yan CG, Wang XD, Zuo XN, Zang YF. DPABI: data processing \& analysis for (resting-state) brain imaging. Neuroinformatics. 2016;14 (3):339-351. doi:10.1007/s12021-016-9299-4

32. Van Dijk KR, Sabuncu MR, Buckner RL. The influence of head motion on intrinsic functional connectivity MRI. Neuroimage. 2012;59(1):431-438. doi:10.1016/j.neuroimage.2011.07.044

33. Noppeney U, Price CJ. Retrieval of visual, auditory, and abstract semantics. Neuroimage. 2002;15(4):917-926. doi:10.1006/nimg.20 01.1016

34. Van EM. The left inferior parietal lobe represents stored hand-postures for object use and action prediction. Front Psychol. 2014;5:333.

35. Ehrenbrusthoff K, Ryan CG, Grüneberg C, et al. The intra- and interobserver reliability of a novel protocol for two-point discrimination in individuals with chronic low back pain. Physiol Meas. 2016;37 (7):1074-1088. doi:10.1088/0967-3334/37/7/1074
36. Middleton FA, Strick PL. Basal ganglia and cerebellar loops: motor and cognitive circuits. Brain Res Rev. 2000;31(2-3):236-250. doi:10.1016/S0165-0173(99)00040-5

37. Tagliazucchi E, Balenzuela P, Fraiman D, Chialvo DR. Brain resting state is disrupted in chronic back pain patients. Neurosci Lett. 2010;485(1):26-31. doi:10.1016/j.neulet.2010.08.053

38. Uwe W, Marco T, Patrick R, Jürgen K, Arno V. Functional and structural correlates of motor speed in the cerebellar anterior lobe. PLoS One. 2014;9(5):e96871. doi:10.1371/journal.pone.0096871

39. Andersen BB. Reduction of Purkinje cell volume in cerebellum of alcoholics. Brain Res. 2004;1007(1):10-18. doi:10.1016/j.brainres. 2004.01.058

40. Visser M, Jefferies E, Embleton KV, Lambon Ralph MA. Both the middle temporal gyrus and the ventral anterior temporal area are crucial for multimodal semantic processing: distortion-corrected fMRI evidence for a double gradient of information convergence in the temporal lobes. $J$ Cogn Neurosci. 2012;24(8):1766-1778. doi:10.1162/jocn_a_00244

41. Xue SW, Li D, Weng XC, Northoff G, Li DW. Different neural manifestations of two slow frequency bands in resting functional magnetic resonance imaging: a systemic survey at regional, interregional, and network levels. Brain Connect. 2014;4(4):242-255. doi:10.1089/brain.2013.0182

\section{Publish your work in this journal}

The Journal of Pain Research is an international, peer reviewed, open access, online journal that welcomes laboratory and clinical findings in the fields of pain research and the prevention and management of pain. Original research, reviews, symposium reports, hypothesis formation and commentaries are all considered for publication. The manuscript management system is completely online and includes a very quick and fair peer-review system, which is all easy to use. Visit http:// www.dovepress.com/testimonials.php to read real quotes from published authors. 